\title{
Classical kinetic energy, quantum fluctuation terms and kinetic-energy functionals
}

\author{
I. P. Hamilton* \\ Department of Chemistry, Wilfrid Laurier University, Waterloo, Canada N2L 3C5. \\ Ricardo A. Mosna đ๋ \\ Instituto de Matemática, Estatística e Computação Científica, \\ Universidade Estadual de Campinas, C.P. 6065, 13083-859, Campinas, SP, Brazil. \\ L. Delle Sit屯 \\ Max-Planck-Institute for Polymer Research, Ackermannweg 10, D 55021 Mainz Germany.
}

\begin{abstract}
We employ a recently formulated dequantization procedure to obtain an exact expression for the kinetic energy which is applicable to all kinetic-energy functionals. We express the kinetic energy of an $N$-electron system as the sum of an $N$-electron classical kinetic energy and an $N$-electron purely quantum kinetic energy arising from the quantum fluctuations that turn the classical momentum into the quantum momentum. This leads to an interesting analogy with Nelson's stochastic approach to quantum mechanics, which we use to conceptually clarify the physical nature of part of the kinetic-energy functional in terms of statistical fluctuations and in direct correspondence with Fisher Information Theory. We show that the $N$-electron purely quantum kinetic energy can be written as the sum of the (one-electron) Weizsäcker term and an $(N-1)$-electron kinetic correlation term. We further show that the Weizsäcker term results from local fluctuations while the kinetic correlation term results from the nonlocal fluctuations. We then write the $N$-electron classical kinetic energy as the sum of the (one-electron) classical kinetic energy and another $(N-1)$-electron kinetic correlation term. For one-electron orbitals (where kinetic correlation is neglected) we obtain an exact (albeit impractical) expression for the noninteracting kinetic energy as the sum of the classical kinetic energy and the Weizsäcker term. The classical kinetic energy is seen to be explicitly dependent on the electron phase and this has implications for the development of accurate orbital-free kineticenergy functionals. Also, there is a direct connection between the classical kinetic energy and the angular momentum and, across a row of the periodic table, the classical kinetic energy component of the noninteracting kinetic energy generally increases as $Z$ increases. Finally, we underline that, although our aim in this paper is conceptual rather than practical, our results are potentially useful for the construction of improved kinetic-energy functionals.
\end{abstract}

Keywords: kinetic-energy functionals, dequantization, Fisher information theory, Nelson's stochastic mechanics, quantum fluctuations, classical kinetic functional

\section{INTRODUCTION}

\section{A. Density Functional Theory}

Density functional theory has developed into an extremely successful approach for the calculation of atomic and molecular properties. [1, 2, 3] In this approach, the electron density, $\rho(\boldsymbol{r})$, is the fundamental variable and properties such as the energy are obtained from $\rho$ rather than from the $N$-electron wavefunction, $\psi\left(\boldsymbol{r}_{1}, \ldots, \boldsymbol{r}_{N}\right)$, as in conventional quantum mechanical approaches based on the Schrödinger equation. The motivation for density functional theory is clear - if properties such as the energy can be obtained from $\rho$ then calculations on systems with a large number of electrons are, in principle, no more difficult than those on systems with a small number. However, this depends on having accurate energy functionals which, in practice, is a serious problem. The energy can be partitioned into kinetic and potential terms and a clear zeroth-order choice of functional for the potential energy is the classical expression $-Z e^{2} \int \frac{\rho(\boldsymbol{r})}{r} d^{3} \boldsymbol{r}+\frac{e^{2}}{2} \iint \frac{\rho\left(\boldsymbol{r}_{1}\right) \rho\left(\boldsymbol{r}_{2}\right)}{r_{12}} d^{3} \boldsymbol{r}_{1} d^{3} \boldsymbol{r}_{2}$. However, for atomic and molecular systems, there is no correspondingly clear zeroth-order choice of functional for the kinetic energy.

\footnotetext{
*Electronic address: ihamilton@wlu.ca

${ }^{\dagger}$ Electronic address: mosna@ime.unicamp.br

${ }^{\ddagger}$ Electronic address: dellsite@mpip-mainz.mpg.de
} 


\section{B. Quantum fluctuations}

One of the key aspects of quantum mechanics is that one cannot simultaneously ascribe well-defined (sharp) values for the position and momentum of a physical system. Motivated by this, quantization procedures have been proposed in which the quantum regime is obtained from the classical regime by adding stochastic terms to the classical equations of motion. In particular, Nelson [5] and earlier work of Fényes [6] and Weizel [7] has shown that the Schrödinger equation can be derived from Newtonian mechanics via the assumption that particles are subjected to Brownian motion with a real diffusion coefficient. The Brownian motion results in an osmotic momentum and adding this term to the classical momentum results in the quantum momentum.

We recently proposed [8] a dequantization procedure whereby the classical regime is obtained from the quantum regime by stripping these "quantum fluctuations" from the quantum momentum resulting in the classical momentum. In particular, we introduced deformations of the momentum operator, which correspond to generic fluctuations of the particle's momentum. These lead to a deformed kinetic energy, which roughly quantifies the amount of "fuzziness" caused by these fluctuations. We showed that the deformed kinetic energy possesses a unique minimum, which is seen to be the classical kinetic energy. In this way, a variational procedure determines the particular deformation that has the effect of suppressing the quantum fluctuations, resulting in dequantization of the system. From this variational procedure we obtain a term (identical to the osmotic momentum of Nelson [5]) which, when added to the classical momentum results in the quantum momentum. This is an interesting point which is further clarified in this paper: the classical limit of the physics of electrons, with its usual statistical interpretation, finds a direct correspondence with our dequantization procedure as we show later.

In this paper we obtain an expression of the quantum-classical correspondence for the kinetic energy when $\rho$ is the fundamental variable for the quantum terms. In this expression the kinetic energy of an $N$-electron system is written as the sum of an $N$-electron classical kinetic energy and an $N$-electron purely quantum kinetic energy arising from the quantum fluctuations that turn the classical momentum into the quantum momentum, as in Nelson's stochastic approach to quantum mechanics. [5] We establish a connection between the osmotic momentum of Nelson, the Weizsäcker term and the Fisher Information. For one-electron orbitals we then obtain an expression for the noninteracting kinetic energy as the sum of the classical kinetic energy and the Weizsäcker term. The Weizsäcker term is well-known and the classical kinetic energy is related to the Thomas-Fermi term which is also well-known. However, we believe that our derivation, which obtains both these terms within a single theoretical framework, is novel. Also, there are significant differences between the classical kinetic energy and the Thomas-Fermi term. In particular, the classical kinetic energy is explicitly dependent on the electron phase. Our expression is therefore at best order $N^{3}$ and can have no practical advantage over the standard Kohn-Sham expression. However, our expression is exact and we will show that it correctly reduces to the Thomas-Fermi term for the uniform electron gas and to the Weizsäcker term for the hydrogen atom. By examining our expression for basis functions that are the product of radial functions and spherical harmonics, we establish a direct connection between the classical kinetic energy and the angular momentum. We believe that this intrinsic connection between the angular momentum and a component of the noninteracting kinetic energy is of significant conceptual value in showing the information that should be incorporated in any kinetic-energy functional.

\section{KINETIC-ENERGY FUNCTIONALS}

We begin by considering some previously proposed kinetic-energy functionals whereby the kinetic energy is obtained from the electron density, $\rho$. Here the electron density is given in terms of the (normalized) wavefunction by

$$
\rho(\boldsymbol{r})=N \int\left|\psi\left(\boldsymbol{r}, \ldots, \boldsymbol{r}_{N}\right)\right|^{2} d^{3} \boldsymbol{r}_{2} \ldots d^{3} \boldsymbol{r}_{N}
$$

so that $\int \rho(\boldsymbol{r}) d^{3} \boldsymbol{r}=N$.

\section{A. Thomas-Fermi and Weizsäcker terms}

A well-known functional for the kinetic energy, formulated by Thomas and Fermi [9, 10], is

$$
T_{T F}=\frac{3 \hbar^{2}}{10 m}\left(3 \pi^{2}\right)^{2 / 3} \int \rho(\boldsymbol{r})^{5 / 3} d^{3} \boldsymbol{r} .
$$


This expression is exact for the uniform electron gas (an $N=\infty$ system) for which the reduced gradient $\left(|\nabla \rho| / 2 k_{f} \rho\right.$ with $\left.k_{f}=\left(3 \pi^{2} \rho\right)^{1 / 3}\right)$ is zero. Another well-known kinetic-energy functional, formulated by Weizsäcker [4] , is

$$
T_{W}=\frac{\hbar^{2}}{8 m} \int \frac{|\nabla \rho(\boldsymbol{r})|^{2}}{\rho(\boldsymbol{r})} d^{3} \boldsymbol{r}
$$

This expression is exact for the ground state of the hydrogen atom (an $N=1$ system).

For atomic systems it might be hoped that an accurate kinetic energy functional could be obtained via some combination of $T_{T F}$ and $T_{W}$ and, in fact, Weizsäcker had proposed $T_{T F}+T_{W}$. Other researchers subsequently proposed either a smaller coefficient for $T_{T F}[11,12,13,14,15]$ or, more commonly, $T_{W}$. A second-order gradient expansion of the density for a nonuniform electron gas (valid for small reduced gradient) leads to the coefficient $\frac{1}{9}$. [16, 17, 18 Other expressions for the kinetic energy have been developed and, of particular relevance to our paper, Herring [19] proposes $T_{\theta}+T_{W}$ where $T_{\theta}$ is termed the relative-phase energy. In our expression for the kinetic energy the relative-phase energy is replaced by the classical kinetic energy.

For large $Z$ atoms, the electron density is slowly varying for the bulk of the electrons in the intermediate $r$ region, a second-order gradient expansion is valid, and the expression $T_{T F}+\frac{1}{9} T_{W}$ (with the Dirac exchange functional [21]) is accurate. However, this expression is not accurate for small and large $r$. [22] For small $r$ the Scott correction, [20] can be employed but for large $r$ no correction is known. Unfortunately, the large $r$ region is (by virtue of the valence electrons) responsible for chemical bonding and Thomas-Fermi theory cannot describe molecular systems. An expression for the kinetic energy which is accurate for large $r$ and which might, in principle, be employed to correct the Thomas-Fermi expression in the large $r$ region would therefore be of significant interest.

The Fisher information, 23, 24] which was developed in information theory as a measure of spatial localization, is given by

$$
\mathscr{I}=\int \frac{|\nabla p(\boldsymbol{r})|^{2}}{p(\boldsymbol{r})} d^{3} \boldsymbol{r}
$$

where $p\left(\boldsymbol{r}_{1}\right)=\int\left|\psi\left(\boldsymbol{r}_{1}, \ldots, \boldsymbol{r}_{N}\right)\right|^{2} d^{3} \boldsymbol{r}_{2} \ldots d^{3} \boldsymbol{r}_{N}$ is the one-electron (probability) density, so that $\rho(\boldsymbol{r})=N p(\boldsymbol{r})$. It follows that $T_{W}=\frac{N \hbar^{2}}{8 m} \mathscr{I}$ and these quantities are essentially identical.

\section{B. Hohenberg-Kohn theorems and Kohn-Sham approach}

Density functional theory was placed on a solid foundation by the work of Hohenberg and Kohn [25] who proved that the total energy can indeed be obtained as a functional of $\rho$. Their proof also applies to the kinetic energy but they could provide no prescription for constructing the exact kinetic-energy functional. Kohn and Sham [26] subsequently provided a prescription for calculating the noninteracting kinetic energy by adapting aspects of Hartree-Fock theory. In Hartree-Fock theory the wavefunction is approximated as the product of $N$ one-electron orbitals (antisymmetrized to ensure that electron exchange is incorporated exactly for the approximate wavefunction). In constructing these orbitals the effect of the other electrons is included only in an average way (through the use of an effective potential) and electron correlation is neglected. Calculations scale as $N^{3}$ and post Hartree-Fock approaches incorporating electron correlation (required for chemical accuracy) typically scale as $N^{5}$ or $N^{7}$. Kohn and Sham employed the orbital approximation but chose the effective potential such that for the one-electron orbitals, $\phi_{i}$, the resulting density is equal to $\rho$. From these orbitals they obtained the noninteracting kinetic energy as $T_{s}=\frac{\hbar^{2}}{2 m} \int \sum_{i=1}^{N}\left|\nabla \phi_{i}\right|^{2} d^{3} \boldsymbol{r}$ rather than as a direct functional of $\rho$. As in Hartree-Fock theory, electron exchange is incorporated exactly and electron correlation is neglected. Complete calculations employ an exchange-correlation functional for the difference between $T_{s}$ and the exact kinetic energy (and also the difference between the classical electrostatic energy and the exact potential energy). In the canonical implementation (with semi-local approximations to the exchange-correlation potential) calculations scale as $N^{3}$ as in Hartree-Fock theory but, because high-quality exchange-correlation functionals have been developed, chemical accuracy can be realized and it is in this form that density functional theory has been most successful for the calculation of atomic and molecular properties.

Despite the success of the Kohn-Sham approach, there has been continued interest in developing expressions (termed orbital-free kinetic-energy functionals) which obtain the noninteracting kinetic energy, $T_{s}$, as a direct functional of $\rho$. The very practical motivation is that these expressions could be order $N$ and much larger systems would therefore be tractable but chemical accuracy has not yet been realized. A recent study [27] carefully analyzed kinetic-energy functionals of the $T_{T F}+\lambda T_{W}$ form while other recent studies [28, 29] considered the accuracy of various kineticenergy functionals which combine $T_{T F}, T_{W}$ and higher-order gradient expansion terms in more complicated ways. The development of orbital-free kinetic-energy functionals continues to be an active area of research. [30, 31, 32, 33, 34, 35] 


\section{QUANTUM-CLASSICAL CORRESPONDENCE}

Consider, for an $N$-electron system, a local deformation $\boldsymbol{P} \rightarrow \boldsymbol{P}_{\boldsymbol{u}}$ of the quantum momentum operator $\boldsymbol{P}=-i \hbar \boldsymbol{\nabla}$, with [8]

$$
\boldsymbol{P}_{\boldsymbol{u}} \psi=(\boldsymbol{P}-i \boldsymbol{u}) \psi
$$

where all quantities in bold face are $3 N$-dimensional vectors and $\boldsymbol{u}$ is real.

Let

$$
T=\frac{1}{2 m} \int(\boldsymbol{P} \psi)^{*}(\boldsymbol{P} \psi) d^{3 N} \boldsymbol{r}
$$

and

$$
T_{\boldsymbol{u}}=\frac{1}{2 m} \int\left(\boldsymbol{P}_{\boldsymbol{u}} \psi\right)^{*}\left(\boldsymbol{P}_{\boldsymbol{u}} \psi\right) d^{3 N} \boldsymbol{r}
$$

be the kinetic terms arising from $\boldsymbol{P}$ and $\boldsymbol{P}_{\boldsymbol{u}}$, respectively.

We recently showed [8] that extremization of $T_{\boldsymbol{u}}$ with respect to $\boldsymbol{u}$-variations leads to the critical point

$$
\boldsymbol{u}_{c}=-\frac{\hbar}{2} \frac{\nabla p_{N}}{p_{N}}
$$

where $p_{N}\left(\boldsymbol{r}_{1}, \ldots, \boldsymbol{r}_{N}\right)=\left|\psi\left(\boldsymbol{r}_{1}, \ldots, \boldsymbol{r}_{N}\right)\right|^{2}$ is the $N$-electron (probability) density (with $\int p_{N} d^{3} \boldsymbol{r}_{1} \cdots d^{3} \boldsymbol{r}_{N}=1$ ). We previously [36] obtained the same expression for $\boldsymbol{u}_{c}$ via a Witten deformation of the quantum momentum. This value of $\boldsymbol{u}_{c}$ results in the classical momentum operator [8, 36]

$$
\boldsymbol{P}_{c} \psi=\left(\boldsymbol{P}+\frac{i \hbar}{2} \frac{\boldsymbol{\nabla} p_{N}}{p_{N}}\right) \psi .
$$

Thus our dequantization procedure automatically identifies the expression for $\boldsymbol{u}_{c}$ (cf Eq. (8)) which when added to the quantum momentum results in the classical momentum. Here $-\boldsymbol{u}_{c}$ is identical to the osmotic momentum of Nelson [5], and adding $-\boldsymbol{u}_{c}$ to the classical momentum results in the quantum momentum.

This value of $\boldsymbol{u}_{c}$ results in

$$
T_{\boldsymbol{u}_{c}}=T-\frac{\hbar^{2}}{8 m} \mathscr{I}_{N},
$$

where $\mathscr{I}_{N}$ is the $N$-electron Fisher information [23]

$$
\mathscr{I}_{N}=\int \frac{\left(\nabla p_{N}\right)^{2}}{p_{N}} d^{3 N} \boldsymbol{r} .
$$

If the wavefunction is written as $\psi=\sqrt{p_{N}} e^{i S_{N} / \hbar}$ where $S_{N}\left(\boldsymbol{r}_{1}, \ldots, \boldsymbol{r}_{N}\right)$ is the $N$-electron phase then a straightforward calculation shows that the action of $\boldsymbol{P}_{c}$ on $\psi$ is given by

$$
\boldsymbol{P}_{c} \psi=\nabla S_{N} \psi
$$

so that, from Eq. (7),

$$
T_{\boldsymbol{u}_{c}}=\frac{1}{2 m} \int p_{N}\left|\nabla S_{N}\right|^{2} d^{3 N} \boldsymbol{r} .
$$

This quantity is the mean kinetic energy of a classical ensemble, described by the density $p_{N}$ and momentum $\nabla S_{N}$ [37, 38] and we therefore refer to $T_{\boldsymbol{u}_{c}}$ as the $N$-electron classical kinetic energy $T_{C l, N}$. 


\section{RESULTS AND DISCUSSION}

The $N$-electron kinetic energy can be expressed, from Eq. (10), as

$$
T_{N}=T_{C l, N}+\frac{\hbar^{2}}{8 m} \mathscr{I}_{N}
$$

This is the sum of the $N$-electron classical kinetic energy and a purely quantum term which is essentially given by the $N$-electron Fisher information although, as our approach is restricted to scalar particles, effects due to electron spin are not explicitly included and our expressions are valid only for a single-spin wavefunction.

We first consider the $N$-electron classical kinetic energy of Eq. (14). It immediately follows from Eq. (13) that $T_{C l, N}=0$ if and only if the $N$-electron phase is constant. Since a constant $N$-electron phase can always be redefined to be zero, this is the case if and only if the wavefunction is real.

We now consider the purely quantum term of Eq. (14). As in Ref. [39] we decompose the $N$-electron density as

$$
p_{N}\left(\boldsymbol{r}_{1}, \ldots, \boldsymbol{r}_{N}\right)=p\left(\boldsymbol{r}_{1}\right) f\left(\boldsymbol{r}_{2}, \ldots, \boldsymbol{r}_{N} \mid \boldsymbol{r}_{1}\right)
$$

where

$$
p\left(\boldsymbol{r}_{1}\right)=\int p_{N}\left(\boldsymbol{r}_{1}, \ldots, \boldsymbol{r}_{N}\right) d^{3} \boldsymbol{r}_{2} \cdots d^{3} \boldsymbol{r}_{N}
$$

and

$$
f\left(\boldsymbol{r}_{2}, \ldots, \boldsymbol{r}_{N} \mid \boldsymbol{r}_{1}\right)=\frac{p_{N}\left(\boldsymbol{r}_{1}, \ldots, \boldsymbol{r}_{N}\right)}{p\left(\boldsymbol{r}_{1}\right)}
$$

In this way, while $p$ is the (already introduced) one-electron probability density, the quantity $f\left(\boldsymbol{r}_{2}, \ldots, \boldsymbol{r}_{N} \mid \boldsymbol{r}_{1}\right)$ is a conditional density in that it represents the electron density associated with a set of values for $\boldsymbol{r}_{2}, \ldots, \boldsymbol{r}_{N}$ given a fixed value for $\boldsymbol{r}_{1}$. Here $p$ and $f$ satisfy the normalization conditions

$$
\begin{gathered}
\int p\left(\boldsymbol{r}_{1}\right) d^{3} \boldsymbol{r}_{1}=1 \\
\int f\left(\boldsymbol{r}_{2}, \ldots, \boldsymbol{r}_{N} \mid \boldsymbol{r}_{1}\right) d^{3} \boldsymbol{r}_{2} \cdots d^{3} \boldsymbol{r}_{N}=1 \quad \forall \boldsymbol{r}_{1} .
\end{gathered}
$$

This immediately yields an expression for the minimizing momentum fluctuations (cf Eq. (8)) as

$$
-\boldsymbol{u}_{c}=\frac{\hbar}{2}\left(\frac{\boldsymbol{\nabla}_{\boldsymbol{r}_{1}} \rho\left(\boldsymbol{r}_{1}\right)}{\rho\left(\boldsymbol{r}_{1}\right)}+\sum_{i=2}^{N} \frac{\boldsymbol{\nabla}_{\boldsymbol{r}_{i}} f\left(\boldsymbol{r}_{2}, \ldots, \boldsymbol{r}_{N} \mid \boldsymbol{r}_{1}\right)}{f\left(\boldsymbol{r}_{2}, \ldots, \boldsymbol{r}_{N} \mid \boldsymbol{r}_{1}\right)}\right),
$$

where the relation $\rho(\boldsymbol{r})=N p(\boldsymbol{r})$ was used. In Eq. (17) it is implicitly assumed that $\rho$ and $f$ result from the same wavefunction and that all necessary representability conditions are therefore satisfied. From Eq. (17) it is possible to distinguish a local part of the momentum fluctuation, $\frac{\hbar}{2} \frac{\boldsymbol{\nabla}_{\boldsymbol{r}_{1}} \rho\left(\boldsymbol{r}_{1}\right)}{\rho\left(\boldsymbol{r}_{1}\right)}$, corresponding to fluctuation of the oneelectron density in the (arbitrary but fixed) variable $\boldsymbol{r}_{1}$, and a nonlocal part, $\frac{\hbar}{2} \sum_{i=2}^{N} \frac{\boldsymbol{\nabla}_{\boldsymbol{r}_{i}} f\left(\boldsymbol{r}_{2}, \ldots, \boldsymbol{r}_{N} \mid \boldsymbol{r}_{1}\right)}{f\left(\boldsymbol{r}_{2}, \ldots, \boldsymbol{r}_{N} \mid \boldsymbol{r}_{1}\right)}$, corresponding to fluctuation of the correlation function $f\left(\boldsymbol{r}_{2}, \ldots, \boldsymbol{r}_{N} \mid \boldsymbol{r}_{1}\right)$.

The $N$-electron Fisher information (cf Eq. (11)) can be written as

$$
\mathscr{I}_{N}=N \int \frac{\left[\boldsymbol{\nabla}_{\boldsymbol{r}_{1}} p_{N}\left(\boldsymbol{r}_{1}, \ldots, \boldsymbol{r}_{N}\right)\right]^{2}}{p_{N}\left(\boldsymbol{r}_{1}, \ldots, \boldsymbol{r}_{N}\right)} d^{3} \boldsymbol{r}_{1} \cdots d^{3} \boldsymbol{r}_{N} .
$$

The decomposition for $p_{N}$ in Eq. (15) can then be used to express this quantity in a more illuminating form as

$$
\begin{aligned}
\mathscr{I}_{N} & =N \int \frac{\left[\boldsymbol{\nabla}_{\boldsymbol{r}_{1}} p\left(\boldsymbol{r}_{1}\right) f\left(\boldsymbol{r}_{2}, \ldots, \boldsymbol{r}_{N} \mid \boldsymbol{r}_{1}\right)+p\left(\boldsymbol{r}_{1}\right) \boldsymbol{\nabla}_{\boldsymbol{r}_{1}} f\left(\boldsymbol{r}_{2}, \ldots, \boldsymbol{r}_{N} \mid \boldsymbol{r}_{1}\right)\right]^{2}}{p\left(\boldsymbol{r}_{1}\right) f\left(\boldsymbol{r}_{2}, \ldots, \boldsymbol{r}_{N} \mid \boldsymbol{r}_{1}\right)} d^{3} \boldsymbol{r}_{1} \cdots d^{3} \boldsymbol{r}_{N} \\
& =N \int \frac{\left[\boldsymbol{\nabla}_{\boldsymbol{r}_{1}} p\left(\boldsymbol{r}_{1}\right)\right]^{2}}{p\left(\boldsymbol{r}_{1}\right)} d^{3} \boldsymbol{r}_{1}+N \int p\left(\boldsymbol{r}_{1}\right) \frac{\left[\boldsymbol{\nabla}_{\boldsymbol{r}_{1}} f\left(\boldsymbol{r}_{2}, \ldots, \boldsymbol{r}_{N} \mid \boldsymbol{r}_{1}\right)\right]^{2}}{f\left(\boldsymbol{r}_{2}, \ldots, \boldsymbol{r}_{N} \mid \boldsymbol{r}_{1}\right)} d^{3} \boldsymbol{r}_{1} \cdots d^{3} \boldsymbol{r}_{N},
\end{aligned}
$$


where Eqs. (16) were used to simplify the first term and cancel the mixed term. We then have

$$
\mathscr{I}_{N}=\int \frac{|\nabla \rho(\boldsymbol{r})|^{2}}{\rho(\boldsymbol{r})} d^{3} \boldsymbol{r}+\int \rho(\boldsymbol{r}) \mathscr{I}_{\mathrm{one}}^{f}(\boldsymbol{r}) d^{3} \boldsymbol{r},
$$

where

$$
\mathscr{I}_{\text {one }}^{f}(\boldsymbol{r})=\int \frac{\left[\boldsymbol{\nabla}_{\boldsymbol{r}_{1}} f\left(\boldsymbol{r}_{2}, \ldots, \boldsymbol{r}_{N} \mid \boldsymbol{r}\right)\right]^{2}}{f\left(\boldsymbol{r}_{2}, \ldots, \boldsymbol{r}_{N} \mid \boldsymbol{r}\right)} d^{3} \boldsymbol{r}_{2} \ldots d^{3} \boldsymbol{r}_{N}
$$

Thus Eq. (19) decomposes the $N$-electron Fisher information as a sum of two terms. The first is local, and is $N$ times $\mathscr{I}$ (cf Eq. (44)), and the second is nonlocal and comprises many-electron effects through $\mathscr{I}_{\text {one. }}^{f}$. This equation provides a connection between the osmotic momentum of Nelson, the Weizsäcker term and the Fisher Information.

\section{A. One-electron kinetic energy}

From Eqs. (3), (14) and (19), we obtain the $N$-electron kinetic energy as

$$
T_{N}=T_{C l, N}+T_{W}+\frac{\hbar^{2}}{8 m} \int \rho(\boldsymbol{r}) \mathscr{I}_{\mathrm{one}}^{f}(\boldsymbol{r}) d^{3} \boldsymbol{r} .
$$

Eq. (20) decomposes the $N$-electron kinetic energy as the sum of a classical term and two purely quantum terms and constitutes an expression of the quantum-classical correspondence for the $N$-electron kinetic energy when $\rho$ is the fundamental variable for the quantum terms.

Each term of Eq. (20) adds an independent nonnegative contribution to the kinetic energy and this equation agrees with the decomposition of Sears et al. [39] when the $N$-electron phase is constant (since $T_{C l, N}$ is zero in this case, as discussed above). Thus we see that the classical term in Eq. (20) improves the lower bound for the general case in which the $N$-electron phase is not constant.

In Eq. (20) the first quantum term contributes to the noninteracting kinetic energy and the second contributes to the kinetic correlation. We now assume that the $N$-electron classical kinetic energy, $T_{C l, N}$, can be decomposed as the sum of a term, $T_{C l}$, which contributes to the noninteracting classical kinetic energy, and a term, $T_{C l}^{c o r r}$, which contributes to the classical kinetic correlation. Terms that contribute to the noninteracting kinetic energy can be estimated by employing the orbital approximation. If the one-electron orbital is written as $\phi_{i}=\sqrt{p} e^{i S_{i} / \hbar}$ where $S_{i}(\boldsymbol{r})$ is the electron phase then $T_{C l}=\frac{1}{2 m} \int p(\boldsymbol{r}) \sum_{i=1}^{N}\left|\boldsymbol{\nabla} S_{i}(\boldsymbol{r})\right|^{2} d^{3} \boldsymbol{r}$ but we have no explicit expression for $T_{C l}^{c o r r}$. From Eq. (20), we then obtain the (one-electron) kinetic energy as

$$
T=T_{C l}+T_{C l}^{c o r r}+T_{W}+\frac{\hbar^{2}}{8 m} \int \rho(\boldsymbol{r}) \mathscr{I}_{\text {one }}^{f}(\boldsymbol{r}) d^{3} \boldsymbol{r} .
$$

\section{B. Weizsäcker term, kinetic correlation term, and quantum fluctuations}

In Eq. (21) the purely quantum terms, $T_{W}$ and $\frac{\hbar^{2}}{8 m} \int \rho(\boldsymbol{r}) \mathscr{I}_{\text {one }}^{f}(\boldsymbol{r}) d^{3} \boldsymbol{r}$, comprise the $N$-electron Weizsäcker term and, as discussed above, arise in our approach from the fluctuations that turn the classical momentum into the quantum momentum, as in Nelson's stochastic approach to quantum mechanics. [5] Many decompositions of the $N$-electron Weizsäcker term are possible [49, 50] and, as noted above, a decomposition similar to ours has previously been proposed [39]. The novelty of our decomposition is that, from the calculation leading to Eq. (18), we can unequivocally identify $T_{W}$ as resulting from the local part of the quantum fluctuations, and $\frac{\hbar^{2}}{8 m} \int \rho(\boldsymbol{r}) \mathscr{I}_{\text {one }}^{f}(\boldsymbol{r}) d^{3} \boldsymbol{r}$ as resulting from the nonlocal part (cf Eq. (17) and the discussion following it). The latter term contributes to the kinetic correlation and we note that an analytic expression for the electron correlation which incorporates both kinetic and Coulombic terms has been proposed. [51] As noted above, $T_{W}$ (or $\mathscr{I}$, which is a measure of spatial localization) has been universally utilized to construct kinetic-energy functionals and has also been employed to characterize electronic properties [52, 53]. By also employing the Shannon entropy power 54|, which is a measure of spatial delocalization, it has been possible to partially characterize many-electron effects [55, 56]. However, the connection between the kinetic correlation term and nonlocal quantum fluctuations provides a new rationale for the need to incorporate this term in exchange-correlation functionals in order to capture the complete range of many-electron effects. 


\section{Noninteracting kinetic energy}

In the orbital approximation kinetic correlation is neglected and omitting these terms in Eq. (21), we obtain the noninteracting kinetic energy as

$$
T_{s}=T_{C l}+T_{W} .
$$

As we can see from the discussion preceding Eq. (21), $T_{C l}$ is linked to the electron phase and there are two limiting cases where this expression is known analytically. For the ground state of the hydrogen atom (an $N=1$ system), the electron phase is zero, so that $T_{C l}=0$. Therefore, $T_{s}=T_{W}$ in this limit. For the uniform electron gas $($ an $N=\infty$ system) the electrons are noninteracting, so that Eq. (22) also applies. Since the distribution is uniform, $T_{W}=0$ in this case and $T_{C l}=T_{s}$ can be calculated, as usual, by adding up the kinetic energies of one-electron orbitals approximated as local plane waves, which results in the Thomas-Fermi term. 18]

The standard expression for the noninteracting kinetic energy (see section [IB and Eq. (26) of Ref. [19]) is $T_{s}=\frac{\hbar^{2}}{2 m} \int \sum_{i=1}^{N}\left|\nabla \phi_{i}\right|^{2} d^{3} \boldsymbol{r}$. In Eq. (27) of Ref. [19], Herring then defines angular variables representing points on the surface of an $N$-dimensional unit sphere as (in our notation) $u_{i}(\boldsymbol{r})=\phi_{i} / \rho^{1 / 2}$. In Eq. (28) of Ref. [19], he then expresses the noninteracting kinetic energy as $T_{s}=T_{\theta}+T_{W}$ where $T_{\theta}$, which is dependent on the $u_{i}$, is termed the relative-phase energy. Comparison of Eq. (22) in this paper and Eq. (28) of Ref. [19] shows that (in the orbital approximation) $T_{C l}$ and $T_{\theta}$ are equivalent. Herring interprets the relative-phase energy as the additional kinetic energy resulting from the exclusion principle which requires the $N$-electron phase to vary with position (when there is more than one electron with the same spin). His results for a variety of one-dimensional potentials show that $T_{\theta}$ is usually a significant fraction of the kinetic energy and that $T_{\theta}$ generally becomes larger relative to $T_{W}$ as $Z$ increases. [19] The contribution of the electron phase to the kinetic energy, which is implicit in hydrodynamic formulations of quantum mechanics, 40] has been noted in other contexts. 19, 41, 42] For hydrogenic orbitals there is an explicit relationship between the electron phase and the angular momentum and for hydrogenic orbitals with nonzero angular momentum, $T_{C l}$ is a significant fraction of the kinetic energy (as shown below). If hydrogenic orbitals are used as basis functions for the ground states of multi-electron atoms then, as $Z$ increases, the exclusion principle will force electrons into orbitals with higher angular momentum and the number of electrons with a given angular momentum will increase in a stepwise fashion. We note that this behavior has been demonstrated for the Thomas-Fermi electron density 43, 44 and there have been several approaches which include angular momentum effects in Thomas-Fermi theory. [45, 46] In the work of Englert and Schwinger [47, 48], angular momentum effects are included for the express purpose of correcting the Thomas-Fermi electron density for large $r$.

Our expression for the noninteracting kinetic energy, $T_{s}=T_{C l}+T_{W}$, is exact and requires no additional proof. However, to gain insight into the nature of $T_{C l}$, we now examine our expression for basis functions that are the product of radial functions and spherical harmonics (here the noninteracting kinetic energy is simply the kinetic energy and Eq. (22) becomes $T=T_{C l}+T_{W}$ ). These basis functions are typically used to represent one-electron orbitals for the ground states of multi-electron atoms. For practical reasons they are usually Slater orbitals but, for simplicity, we present results for hydrogenic orbitals. We explicitly show that, for these basis functions, our expression for the kinetic energy is correct and furthermore, that it is correct for the radial distributions of the integrands of $T, T_{C l}$ and $T_{W}$. That is, that for each value of $r$, the integrand of $T$ is equal to the sum of the integrands of $T_{C l}$ and $T_{W}$. The hydrogenic orbitals, $\psi(n, l, m)$, are dependent on the principal quantum number $n$, the angular momentum quantum number $l$ and the magnetic quantum number $m$ but the total energy is dependent only on $n$ and is (in atomic units) $E=-1 / 2 n^{2}$. Then, from the virial expression for Coulombic systems, the kinetic energy is $T=-E=1 / 2 n^{2}$. The classical kinetic energy is zero for $\psi(2,0,0)$ and $\psi(2,1,0)$ and, from direct calculation, $T_{W}$ is $1 / 8$ which is equal to $T$. However, the classical kinetic energy is nonzero for $\psi(2,1,1)$ and $\psi(2,1,-1)$ and, from direct calculation, both $T_{C l}$ and $T_{W}$ are $1 / 16$ and $T_{C l}+T_{W}$ is equal to $T$. Radial distributions (integrated over the angular variables) of the integrands for $T_{C l}, T_{W}$ and $T$ are shown in Fig. 1(a). The radial distribution for $T_{C l}$ is dependent on $n, l$ and $|m|$ but the classical kinetic energy is dependent only on $n$ and $|m|$ and $T_{C l}=\frac{|m|}{n} T=|m| / 2 n^{3}$. Thus $T_{C l}$ is constant for $n$ and $|m|$ fixed and this is illustrated in Fig. 1(b)-(d) which shows the radial distributions for $T_{C l}, T_{W}$ and $T$ for $n$ $=5,|m|=1$ and $l=1$ to 3 . In these three cases the radial distributions for $T_{C l}$ all integrate to $1 / 250$. For $n$ and $l$ fixed, $T_{C l}$ increases from 0 to $l / 2 n^{3}$ as $|m|$ increases from 0 to $l$ and this is illustrated in Fig. 1(e)-(h) which shows the radial distributions for $T_{C l}, T_{W}$ and $T$ for $n=5, l=4$ and $|m|=1$ to 4 . In these four cases the radial distributions for $T$ are identical and in each of Fig. 1(b)-(h) the radial distributions for $T$ integrate to $1 / 50$.

For the ground states of multi-electron atoms we expect that $T_{C l}$ will be greater than zero but smaller than $T_{T F}$ (when the reduced gradient is small $T_{T F}$ has been shown [19, 42] to be an upper bound to $T_{C l}$ ) and, across a row of the periodic table, $T_{C l}$ generally increases as $Z$ increases. For example, the one-electron orbital for the ground state of the $\mathrm{C}$ atom will have a larger $l=1$ contribution than will that for the ground state of the Be atom. Correspondingly, $T_{s}$ for the $\mathrm{C}$ atom will have a larger $T_{C l}$ component than will that for the Be atom. However, we have no algorithm 
for optimizing the $T_{C l}$ component of the one-electron orbital. Since $T_{C l}$ is dependent on the $m$ value for each basis function this algorithm would be, at best, order $N^{3}$ and could have no practical advantage over the standard KohnSham algorithm.

It is important to note that, in our approach, the classical kinetic energy is zero if the orbital is real. Thus, whereas $T_{C l}$ is nonzero for $\psi(2,1,1)$ and $\psi(2,1,-1)$ (with $|m|=1$ ), it is zero for the familiar $p_{x}$ and $p_{y}$ orbitals (formed from their linear combinations). For these real orbitals $T_{W}$ is $1 / 8$ which is equal to $T$ and this is appropriate as, although $m$ is not zero, the expectation value of $L_{z}$ is. To obtain an expression corresponding to $T_{s}=T_{C l}+T_{W}$ it is necessary to partition the Weizsäcker term as $T_{W}=T_{W}^{\phi}+T_{W}^{r, \theta}$ where $T_{W}^{\phi}$ results from local fluctuations in $\phi$ (and corresponds to $T_{C l}$ for $|m|=1$ ) and $T_{W}^{r, \theta}$ results from local fluctuations in $r$ and $\theta$ (as does $T_{W}$ for $|m|=1$, to which it is identical). For the $p_{x}$ and $p_{y}$ orbitals the radial distributions of $T_{W}^{\phi}$ and $T_{W}^{r, \theta}$ are identical to those of $T_{C l}$ and $T_{W}$ in Fig. 1(a). From a practical viewpoint the expressions $T_{C l}+T_{W}$ and $T_{W}^{\phi}+T_{W}^{r, \theta}$ are completely equivalent and are equally useful as decompositions of $T_{s}$ but their interpretation is different.
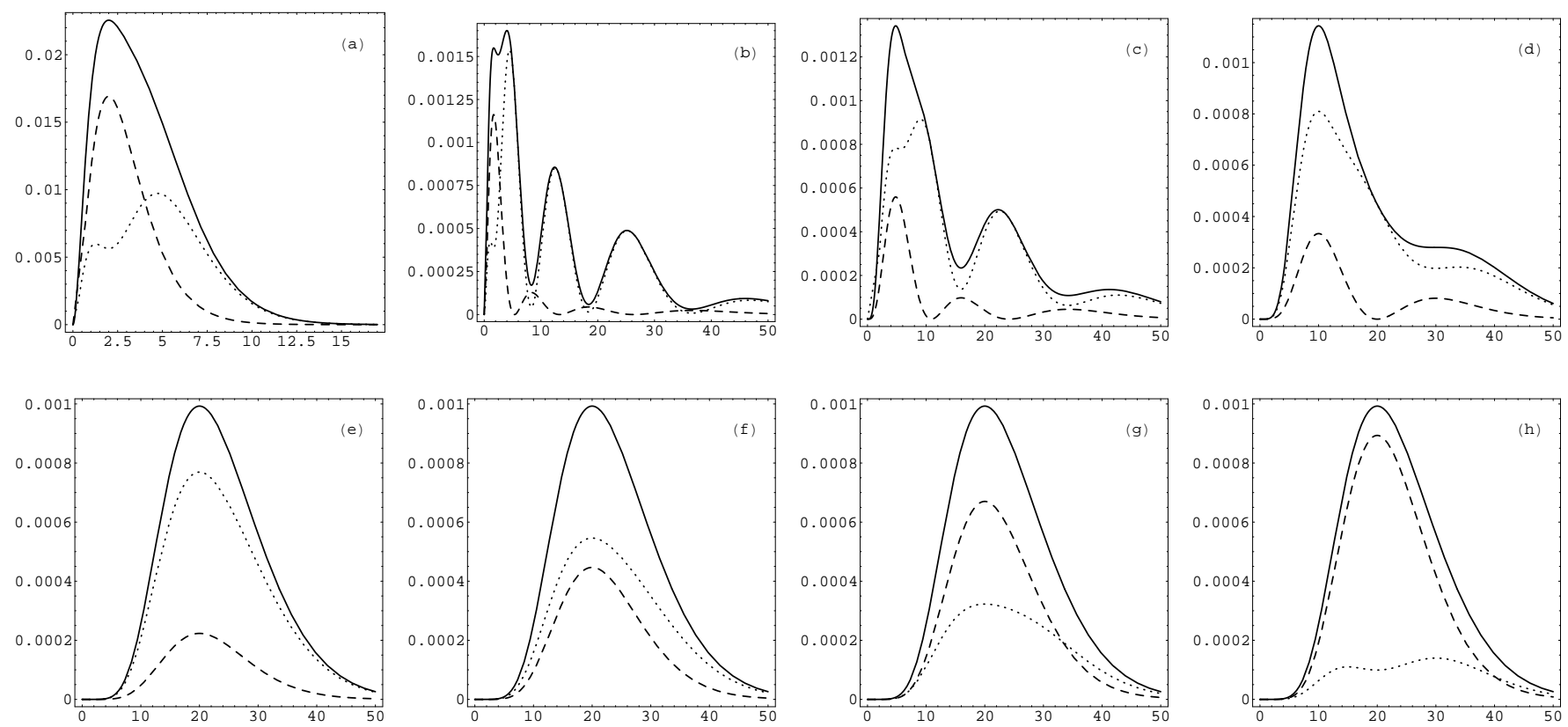

FIG. 1: Radial distributions (integrated over the angular variables) of the integrands for $T_{C l}$ (dashed curve), $T_{W}$ (dotted curve) and $T=T_{C l}+T_{W}$ (solid curve) for hydrogenic orbitals with (a) $n=2, l=1,|m|=1$; (b)-(d) $n=5,|m|=1$ and $l=1$ to 3 ; (e)-(h) $n=5, l=4$ and $|m|=1$ to 4 . The horizontal axis is in atomic units.

\section{CONCLUSIONS}

In this paper we employed a recently formulated dequantization procedure to obtain an exact expression for the kinetic energy which is applicable to all kinetic-energy functionals. In this expression the kinetic energy of an $N$-electron system is written as the sum of the $N$-electron classical kinetic energy and the $N$-electron purely quantum kinetic energy arising from the quantum fluctuations that turn the classical momentum into the quantum momentum. Our dequantization procedure also results in a term (identical to the osmotic momentum of Nelson [5]) which, when added to the classical momentum results in the quantum momentum. We thereby established a connection between Nelson's stochastic approach to quantum mechanics, the Weizsäcker term and the Fisher Information Theory. Moreover, the connection to Fisher Information Theory provides a basis for an interesting conceptual interpretation of some terms contributing to the kinetic-energy functional.

We wrote the $N$-electron purely quantum kinetic energy as the sum of the (one-electron) Weizsäcker term which results from the local quantum fluctuations and a kinetic correlation term which results from the nonlocal quantum correlations. We also wrote the $N$-electron classical kinetic energy as the sum of the (one-electron) classical kinetic energy and another kinetic correlation term. We then obtained an expression for the noninteracting kinetic energy as the sum of the classical kinetic energy and the Weizsäcker term. The Weizsäcker term is well-known and the classical 
kinetic energy is related to the Thomas-Fermi term which is also well-known. However, we believe that our derivation, which obtains both these terms within a single theoretical framework, is novel. Also, there are significant differences between the classical kinetic energy and the Thomas-Fermi term and we conclude with some further remarks on our expression for the noninteracting kinetic energy.

Our expression is exact and we have shown that for the ground state of the hydrogen atom it correctly reduces to the Weizsäcker term while for the uniform electron gas it correctly reduces to the Thomas-Fermi term (which is identical to the classical kinetic energy for this system). However, the classical kinetic energy (unlike the ThomasFermi term) is explicitly dependent on the electron phase. The expression $T_{s}=T_{C l}+T_{W}$ is therefore at best order $N^{3}$ and can have no practical advantage over the standard Kohn-Sham expression. To gain insight into the nature of $T_{C l}$, we examined our expression for basis functions that are the product of radial functions and spherical harmonics and established a direct connection between the classical kinetic energy and the angular momentum. We believe that this intrinsic connection between the angular momentum and a component of the noninteracting kinetic energy is of significant conceptual value in showing the information that should be incorporated in any kinetic-energy functional.

For small and intermediate $Z$ atoms, the basic problem with the expression $T_{s}=T_{T F}+\lambda T_{W}\left(\right.$ or $\left.\lambda T_{T F}+T_{W}\right)$ is that $T_{W}$ incorporates exactly a part of the noninteracting kinetic energy that is also incorporated approximately in $T_{T F}$. [12] This component of $T_{T F}$ should be removed and that is why simply optimizing $\lambda$ offers only limited improvement. 27] The expression $T_{s}=T_{C l}+T_{W}$ is a significant improvement in this regard as $T_{C l}$ and $T_{W}$ are completely independent. However, as the classical kinetic energy is explicitly dependent on the electron phase, our expression is manifestly not orbital-free. As all explicit information regarding the electron phase is lost in constructing the electron density it is clear that any direct functional of $\rho$ which embodies this information must be highly nonlocal. [19, 57, 58, 59, 60] Reconstructing this information from the electron density represents a significant challenge for the development of accurate orbital-free kinetic-energy functionals.

For large $Z$ atoms, the electron density is slowly varying for the bulk of the electrons in the intermediate $r$ region and a second-order gradient expansion is valid. However, this expression is not valid for large $r$. Unfortunately, the large $r$ region is (by virtue of the valence electrons) responsible for chemical bonding and Thomas-Fermi theory cannot describe molecular systems. Our expression is equally valid for intermediate and large $r$ but it is much more difficult to evaluate. For large $Z$ atoms (where the order $N^{3}$ aspect is of greatest concern) it would, in principle, be possible to develop a hybrid approach in which $T_{T F}+\frac{1}{9} T_{W}$ (with the Dirac exchange functional [21]) is employed for the bulk of the electrons in the intermediate $r$ region and corrected for large $r$ by evaluating $T_{C l}+T_{W}$ for the valence electrons only.

\section{Acknowledgments}

IPH acknowledges funding from NSERC and thanks Wilfrid Laurier University for support. RAM acknowledges FAPESP for financial support.

[1] R. G. Parr and W. Yang, Density Functional Theory of Atoms and Molecules, (Oxford University Press, New York, 1989).

[2] R. M. Dreizler and E. K. U. Gross, Density Functional Theory: An Approach to the Quantum Many Body Problem, (Springer-Verlag, Berlin, 1990).

[3] W. Koch and M. C. Holthausen, A Chemist's Guide to Density Functional Theory, (Wiley-VCH, Weinheim, 2000).

[4] C. F. v Weizsäcker, Z. Phys. 96, 431 (1935).

[5] E. Nelson, Phys. Rev. 150, 1079 (1966); E. Nelson, Dynamical Theories of Brownian Motion (Princeton Univ. Press, Princeton, 1967).

[6] I. Fényes, Z. Physik 132, 81 (1952).

[7] W. Weizel, Z. Physik 134, 264 (1953); 135, 270 1953; 136, 582 (1954).

[8] R. A. Mosna, I. P. Hamilton and L. Delle Site, J. Phys. A 39, L229 (2006).

[9] L. H. Thomas, Proc. Camb. Phil. Soc. 23, 542 (1927).

[10] E. Fermi, Rend. Accad. Lincei 6, 602 (1927).

[11] N. H. March and W. H. Young, Proc. Phys. Soc. 72, 182 (1958).

[12] P. K. Acharya, L. J. Bartolotti, S. B. Sears, and R. G. Parr, Proc. Nat. Acad. Sci. 77, 6978 (1980).

[13] J. L. Gázquez and E. V. Ludeña, Chem. Phys. Lett. 83, 145 (1981).

[14] J. L. Gázquez and J. Robles, J. Chem. Phys. 76, 1467 (1982).

[15] P. K. Acharya, J. Chem. Phys. 78, 2101 (1983).

[16] A. S. Kompaneets and E. S. Pavlovski, Sov. Phys.-JETP 4, 328 (1957).

[17] P. A. Kirzhnits, Sov. Phys.-JETP 5, 64 (1957).

[18] W. Yang, Phys. Rev. A 34, 4575 (1986). 
[19] C. Herring, Phys. Rev. A 34, 2614 (1986).

[20] J. M. C. Scott, Philos. Mag. 43, 859 (1952).

[21] P. A. M. Dirac, Proc. Cambridge Philos. Soc. 26, 376 (1930).

[22] L. Spruch, Rev. Mod. Phys. 63, 151 (1991).

[23] R. A. Fisher, Proc. Cambridge Philos. Soc. 22, 700 (1925).

[24] A. Nagy, J. Chem. Phys. 119, 9401 (2003).

[25] P. Hohenberg and W. Kohn, Phys. Rev. 136, B864 (1964).

[26] W. Kohn and L. J. Sham, Phys. Rev. 140, A1133 (1965).

[27] G. K. Chan, A. J. Cohen and N. C. Handy, J. Chem. Phys. 114, 631 (2001).

[28] S. S. Iyengar, M. Ernzerhof, S. N. Maximoff and G. E. Scuseria, Phys. Rev. A 63, 052508 (2001).

[29] F. Tran and T. A. Wesołowski, Chem. Phys. Lett. 360, 209 (2002).

[30] E. Sim, J. Larkin, K. Burke, C. W. Bock, J. Chem. Phys. 118, 8140 (2003).

[31] H. Jiang and W. T. Yang, J. Chem. Phys. 121, 2030 (2004).

[32] J. D. Chai and J. A. Weeks, J. Phys. Chem. B 108, 6870 (2004).

[33] X. Blanc X and E. Cances, J. Chem. Phys. 122, 214106 (2005).

[34] I. V. Ovchinnikov and D. Neuhauser, J. Chem. Phys. 124, 024105 (2006).

[35] B. Zhou and Y. A. Wang, J. Chem. Phys. 124, 081107 (2006).

[36] R. A. Mosna, I. P. Hamilton and L. Delle Site, J. Phys. A 38, 3869 (2005), quant-ph/0504124

[37] H. Goldstein, Classical Mechanics, 2nd ed. (Addison-Wesley, Reading, MA, 1980).

[38] P. R. Holland, The Quantum Theory of Motion (Cambridge University Press, Cambridge, 1993).

[39] S. B. Sears, R. G. Parr and U. Dinur, Isr. J. Chem. 19, 165 (1980).

[40] S. K. Ghosh and B. M. Deb, Phys. Rep. 92, 1 (1982).

[41] S. Luo, J. Phys. A 35, 5181 (2002).

[42] L. Delle Site, J. Phys. A 38, 7893 (2005).

[43] J. H. D. Jensen and J. M. Luttinger, Phys. Rev. 86, 907 (1952).

[44] T. A. Oliphant, Jr., Phys. Rev. 104, 954 (1956).

[45] H. Hellman, Acta Physicochem USSR 4, 225 (1936).

[46] G. Kemister and S. Nordholm, J. Chem. Phys. 76, 5043 (1982).

[47] B.-G. Englert and J. Schwinger, Phys. Rev. A 29, 2339 (1984).

[48] B.-G. Englert and J. Schwinger, Phys. Rev. A 32, 47 (1985).

[49] M. S. Miao, J. Phys. A 34, 8171 (2001).

[50] P. W. Ayers, J. Math. Phys. 46, 062107 (2005).

[51] L. Delle Site, J. Phys. A 39, 3047 (2006).

[52] S. R. Gadre, Adv. Quantum Chem. 22, 1 (1991).

[53] E. Romera and J. S. Dehesa, Phys. Rev. A 50, 256 (1994).

[54] C. E. Shannon, Bell Syst. Tech. J. 27, 623 (1948).

[55] E. Romera and J. S. Dehesa, J. Chem. Phys. 120, 8906 (2004).

[56] R. P. Sagar and N. L. Guevara, J. Chem. Phys. 123, 044108 (2005).

[57] C. Herring and M. Chopra, Phys. Rev. A 37, 31 (1988).

[58] D. J. Lacks and R. G. Gordon, J. Chem. Phys. 100, 4446 (1994).

[59] P. García-González, J. E. Alvarellos and E. Chacón, Phys. Rev. A 54, 1897 (1996).

[60] N. H. March, Int. J. Quantum Chem. 92, 1(2003). 